\title{
Metadata to the MOSAIC database on monitoring of small waters for aquatic invertebrates and agrochemicals
}

Nanina Tron (1), Marlen Heinz \& Stefan Lorenz (1)
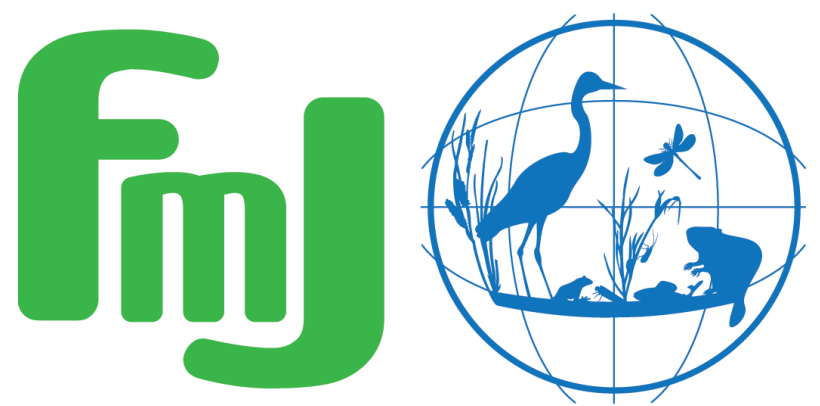

Freshwater Metadata Journal 



\title{
Metadata to the MOSAIC database on monitoring of small waters for aquatic invertebrates and agrochemicals
}

\author{
Nanina Tron ${ }^{1}$ (iD , Marlen Heinz ${ }^{1} \&$ Stefan Lorenz ${ }^{1}$ (iD) \\ 1 Julius Kuehn Institute; Inst. for Ecological Chemistry, Plant Analysis \& Stored Product Protection, Berlin, Germany; corresponding \\ author: stefan.lorenz@julius-kuehn.de
}

Please cite this paper as follows: Tron, N., Heinz, M. \& Lorenz, S., 2019. Metadata to the MOSAIC database on monitoring of small waters for aquatic invertebrates and agrochemicals. Freshwater Metadata Journal 44: 1-7. https://doi.org/10.15504/fmj.2019.44

Received: 2019-04-11 / Published: 2019-05-01

\section{Keywords}

farmland ponds, insect monitoring, invertebrate monitoring, kettle holes, pesticide monitoring, prairie potholes, species distribution, water quality

\section{Short description of the dataset/summary}

The MOSAIC database holds data collected by the Julius Kuehn Institute under its monitoring activities for the implementation of Germany's National Action Plan for the Sustainable Use of Plant Protection Products. At this stage (May 2019), the database contains data on benthic macro-invertebrate community composition and concentrations of pesticides of currently 162 small lentic water bodies ( $<1$ ha surface area) of North-East Germany. MOSAIC will be continuously updated with data from new sampling campaigns and future projects concerning the impacts of agriculture and agrochemicals on the ecological status of lentic and lotic small water bodies. Further sampling locations covering a wider geographical range will be included in future. Sampling locations are situated on or adjacent to agricultural fields and partly at reference sites, and sampling was performed using a multi-habitat-sampling method (invertebrates) and grab or passive sampling of water (pesticides). Taxa were identified to the most precise taxonomic level possible (species level whenever possible). Additionally, the database contains information on physico-chemical parameters, nutrients and agricultural management.

The content of the MOSAIC database presents a unique resource on the biodiversity of Germany's small water bodies and its potential threat by agriculture. At the current stage, it focuses on lentic water bodies which are hot spots of biodiversity. MOSAIC allows extracting information on e.g. toxic pressure of agrochemicals, field crop impact or vegetated buffer strips on all major groups of benthic invertebrates. The access is currently available to JKI staff and collaborating researchers with future web-based open access possibilities planned. 


\section{Short description of the dataset/summary (original/national language)}

Die MOSAIC-Datenbank enthält Daten, die vom Julius Kühn-Institut während seiner Aktivitäten zur Umsetzung des Nationalen Aktionsplans zur nachhaltigen Anwendung von Pflanzenschutzmitteln erhoben wurden. Die Datenbank enthält aktuell (Stand Mai 2019) Daten zur Zusammensetzung der Lebensgemeinschaft benthischer wirbelloser Organismen (Makrozoobenthos) sowie Konzentrationen an Pflanzenschutzmitteln aus 162 stehenden Kleingewässern (Gewässergröße < 1 ha) Nordostdeutschlands. MOSAIC wird mit Daten neuer Untersuchungen sowie zukünftiger Projekte über den Einfluss der Landwirtschaft auf den ökologischen Zustand von Kleingewässern kontinuierlich erweitert werden. Zukünftige Probenahme-Standorte werden eine größere geographische Ausdehnung über Nordostdeutschland hinaus abdecken. Die Untersuchungsgewässer liegen entweder auf oder angrenzend an landwirtschaftlichen Nutzflächen und zum Teil in Referenzgebieten. Die Probenahmen wurden mittels Multi-Habitat-Beprobung (Makrozoobenthos) oder durch Passivsammler und Schöpfproben (Agrarchemikalien) durchgeführt. Die gefundenen Taxa des Makrozoobenthos wurden bis zum bestmöglichen taxonomischen Niveau (wenn möglich Artniveau) bestimmt. Die Datenbank enthält weiterhin Informationen zu chemisch-physikalischen Wasserparametern, Nährstoffbelastungen sowie zum Management der umgebenden landwirtschaftlichen Nutzflächen.

Der Inhalt der MOSAIC-Datenbank stellt eine einzigartige Ressource über die Biodiversität von Deutschlands Kleingewässern und deren potenzielle Bedrohung durch die Landwirtschaft dar. Im gegenwärtigen Stadium konzentriert sich der Inhalt der Datenbank auf stehende Kleingewässer, die zu den Hot Spots der Biodiversität zählen. MOSAIC ermöglicht es, die Auswirkungen z.B. der Toxizität von Agrarchemikalien, des Anbaus verschiedener Feldkulturen oder der Ausgestaltung von Gewässerrandstreifen auf alle wichtigen Gruppen des Makrozoobenthos abzuschätzen. Der Zugang steht derzeit den Mitarbeitern des JKI sowie kooperierenden Wissenschaftlern zur Verfügung, wobei zukünftige webbasierte Open-Access-Möglichkeiten geplant sind.

\section{General information}

dataset entry ID:

name of the dataset:

full name of the dataset:

full name of the datast (original/national language):

Metadaten der MOSAIC Datenbank - Monitoring von Wirbellosen und Agrarchemikalien in Kleingewässern

dataset short name:

type of dataset:

data type:

science keywords according to GCMD:

topic:

SO topic category according to ISO 19115:

INSPIRE keywords according to GEMET:

Farming, Biota, Environment, Inland Waters

Habitats and biotopes, Land use, Species distribution

own science keywords:

related project:

funding: farmland ponds, insect monitoring, invertebrate monitoring, kettle holes, pesticide monitoring, prairie potholes, species distribution, water quality National monitoring of biodiversity in agricultural areas own funding 


\section{Technical and administrative specifications}

$\begin{array}{ll}\begin{array}{l}\text { data format: } \\ \text { others/details: }\end{array} & \text { others/specify } \\ \text { operating system: } & \text { PostgreSQL } \\ \text { data language: } & \text { Win } 8 / 8.1 \\ \text { current access level: } & \text { German } \\ \text { currently available through GBIF: } & \text { restricted access, internal } \\ \text { exchange planned: } & \text { no } \\ \text { data in data repository: } & \text { yes }\end{array}$

Do you plan to publish the data on the Freshwater Biodiversity Data Portal:

$\begin{array}{ll}\text { update level: } & \text { continuously updated } \\ \text { documentation: } & \\ \text { type: } & \text { internal description } \\ \text { language: } & \text { German } \\ & \\ \text { contact details: } & \\ \text { metadata contact person: } & \\ \text { first, last name: } & \text { Nanina Tron } \\ \text { phone: } & \text { 0049 30 8304 2330 } \\ \text { email: } & \text { nanina.tron@julius-kuehn.de } \\ \text { institution: } & \text { Julius Kuehn Institute } \\ \text { address: } & \text { Königin-Luise-Straße 19 } \\ \text { postal code, city: } & 14195 \text { Berlin } \\ \text { province, state: } & \text { Berlin } \\ \text { country } & \text { Germany } \\ \text { web address: } & \text { https://www.julius-kuehn.de/oepv/personal/ } \\ \text { technical contact person: } & \\ \text { first, last name: } & \text { Nanina Tron } \\ \text { phone: } & \text { 0049308304 2330 } \\ \text { email: } & \text { nanina.tron@julius-kuehn.de } \\ \text { scientific contact person: } & \text { Stefan Lorenz } \\ \text { first, last name: } & 00493083042330 \\ \text { phone: } & \\ \text { email: } & \end{array}$

\section{Intellectual property rights and citation}

dataset creator (data compiler):

contact name:

Stefan Lorenz

contact email:

stefan.lorenz@julius-kuehn.de

contact institution:

Julius Kuehn Institute

data contributors to/owners of this dataset:

multiple

number:

3

data contributor/owner 1:

$\begin{array}{ll}\text { contact name: } & \text { Stefan Lorenz } \\ \text { contact email: } & \text { stefan.lorenz@julius-kuehn.de } \\ \text { contact institute: } & \text { Julius Kuehn Institute }\end{array}$


criteria for using this part of the dataset:

The dataset needs to be requested from dataset creator with specific conditions of use.

data contributor/owner 2:

$\begin{array}{ll}\text { contact name: } & \text { Marlen Heinz } \\ \text { contact email: } & \text { marlen.heinz@julius-kuehn.de } \\ \text { contact institute: } & \text { Julius Kuehn Institute }\end{array}$

criteria for using this part of the dataset:

The dataset needs to be requested from dataset creator with specific conditions of use.

data contributor/owner 3:

contact name:

Matthias Stähler

contact email:

matthias.staehler@julius-kuehn.de

contact institute:

Julius Kuehn Institute

criteria for using this part of the dataset:

The dataset needs to be requested from dataset creator with specific conditions of use.

citation of this dataset:

author(s): $\quad$ Stefan Lorenz, Marlen Heinz

title and journal (name, number, pages):

The MOSAIC database on monitoring of small waters for aquatic invertebrates and agrochemicals.

year:

2019

citation of the metadata:

author(s):

Tron N., Heinz M. \& Lorenz S.

title and journal (name, number, pages):

Metadata to the MOSAIC database on monitoring of small waters for aquatic invertebrates and agrochemicals. Freshwater Metadata Journal 44: 1-7

year: 2019

doi: $\quad$ https://doi.org/10.15504/fmj.2019.44

\section{General data specifications}

regional coverage of the dataset:

spatial extent of the dataset:

continents:

regional

countries:

Europe

Europe: Germany

world climatic regions according to Köppen:

Group C: temperate/mesothermal climates

Group D: continental/microthermal climate

freshwater ecoregions of the world (FEOW) according to WWF:

Europe: Central \& Western Europe

European ecoregions according to Illies (WFD):

Central Plains (ER14)

ecosystem type:

covered timeframe:

comments: lakes/ponds, wetlands, general freshwater

$2015-2019$

The covered timeframe will be continuously updated. 


\section{Site specifications}

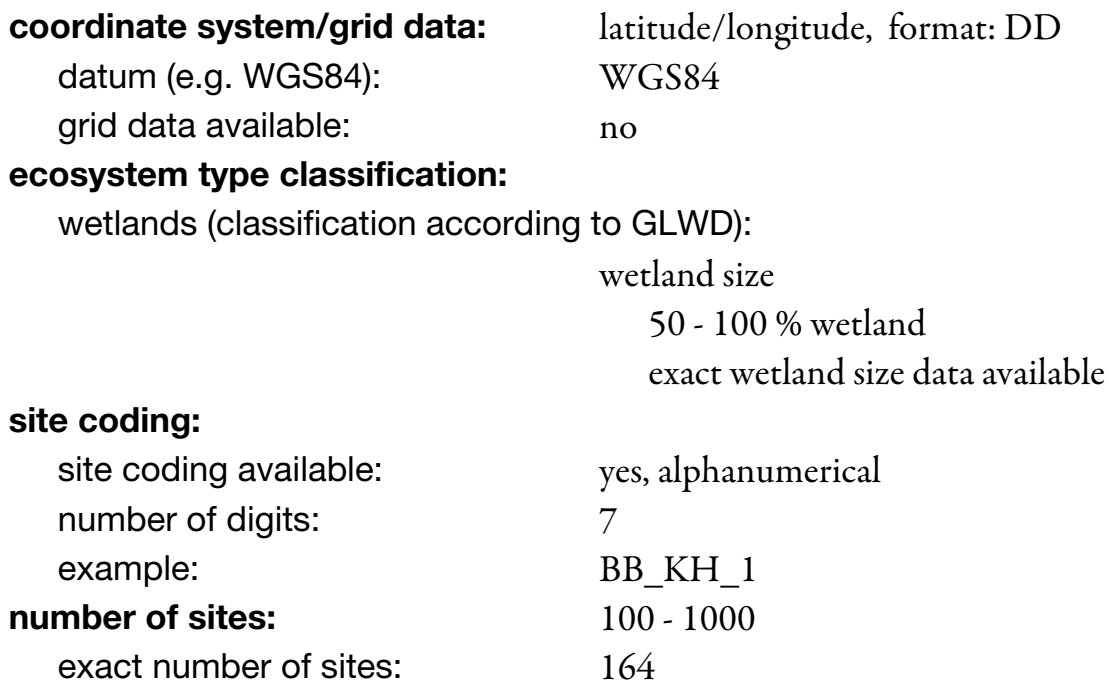

\section{Climate and environmental data}

\section{climate related data: environmental data:}

available parameters per site:

physico-chemical data:

other physico-chemical parameters:

availability of physico-chemical data, if there is more than one sample per site:

stressors influencing the sites:

reference sites available: turbidity, cyanobacteria chlorophyll

no climate data available

no environmental data per catchment available

information on riparian vegetation (incl. information on modification) data source: field mapping substrate composition data source: estimated \% coverage information on instream habitat (incl. information on modification) data source: estimated \% coverage buffer strip width, surrounding field crop, available habitats data source: field mapping ortho $\mathrm{P}$, nitrate, nitrite, ammonium, oxygen content, water temperature, $\mathrm{pH}$, conductivity, chlorophyll mean values per site yes 


\begin{tabular}{|c|c|c|c|c|}
\hline stressor & $\begin{array}{l}\text { restored sites } \\
\text { available }\end{array}$ & $\begin{array}{l}\text { data before/after } \\
\text { restoration } \\
\text { available }\end{array}$ & $\begin{array}{l}\text { stressor gradient } \\
\text { available }\end{array}$ & comments \\
\hline toxic stress & no & no & yes & \\
\hline $\begin{array}{l}\text { hydrologic stress (e.g. } \\
\text { impoundment, flow } \\
\text { velocity reduction, } \\
\text { hydropeaking, water } \\
\text { abstraction, flow } \\
\text { velocity increase, etc.) }\end{array}$ & no & no & no & $\begin{array}{l}\text { permanent and temporary ponds } \\
\text { included }\end{array}$ \\
\hline
\end{tabular}

\section{Biological data}
biological data origin:
general compilation,
habitat-specific quantitative assessment of invertebrates standardised by area using hand nets
organism group addressed: macro-invertebrates (Mollusca, Ephemeroptera, Odonata, Plecoptera, Coleoptera, Trichoptera, Chironomidae)

\section{Sample specifications/sample resolution}

\section{macro-invertebrates: \\ sample information:}

covered timeframe:

$2015-2019$

historical data:

no

palaeo data:

no

season:

spring

temporal resolution/frequency of sampling:

time series data:

per year

comments:

no

\section{taxonomic resolution:}

level:

The covered time frame is continuously amended.

percentage of species level data: 80

\section{taxonomic coding:}

taxalist according to:

\section{AQEM/STAR}

reference(s):

family, sub-family, genus, species

Schmidt-Kloiber, A., Graf, W., Lorenz, A. \& Moog, O. (2006): The

AQEM/STAR taxalist - a pan-European macro-invertebrate ecological database and taxa inventory. Hydrobiologia 566: 325-342.

\section{sample specifications:}

type:

quantitative (abundance data)

replicate samples:

yes

number of samples:

320

specification of method(s) used for sampling and sorting:

Habitat specific sampling with hand nets. Each habitat with a percent share

$>10 \%$ of all available habitats was sampled in 3 replicates. A minimum area of

$0.6 \mathrm{~m}^{2}$ was sampled.

sample type (e.g. habitat specific samples, composite samples etc.): 
habitat specific samples

specific sample location (e.g. littoral, profundal, transect, shoreline, hyporheic zone, etc.):

littoral

\section{Other specifications}

GIS layers, shape files related to the dataset:

no data available

availability of photos:

yes

quality control procedures:

Were any quality control procedures applied to your dataset?

yes

quality control protocols and comments:

Samples were almost exclusively sent to taxonomic experts for the respective macro-invertebrate groups for identification (e.g. Institute BIOTA, X.-F. Garcia, M. Brauns, T. Frase, S. Speth, C.-J. Otto).

\section{Acknowledgements}

We thank Dominique Conrad, Anne Köppen, Manuel König, Christine Reichmann, Gabi Smykalla, Ina Stachewicz, Agnes Wolf and Elke Zeidler for their valuable work in providing these data. 